\title{
A Supervised Artificial Immune Classifier for Remote-Sensing Imagery
}

\author{
Yanfei Zhong, Liangpei Zhang, Member, IEEE, Jianya Gong, and Pingxiang Li, Member, IEEE
}

\begin{abstract}
The artificial immune network (AIN), which is a new computational intelligence model based on artificial immune systems inspired by the vertebrate immune system, has been widely utilized for pattern recognition and data analysis. However, due to the inherent complexity of current AIN models, their application to remote-sensing image classification has been rather limited. This paper presents a novel supervised classification algorithm based on a multiple-valued immune network, which is a novel AIN model, to perform remote-sensing image classification. The proposed method trains the immune network using the samples of regions of interest and obtains an immune network with memory to classify the remote-sensing imagery. Two experiments with different types of images are performed to evaluate the performance of the proposed algorithm in comparison with other traditional image classification algorithms: Parallelepiped, Minimum Distance, Maximum Likelihood, and Back-Propagation Neural Network. The results evince that the proposed algorithm consistently outperforms the traditional algorithms in all the experiments and, hence, provides an effective option for processing remote-sensing imagery.
\end{abstract}

Index Terms-Artificial immune systems (AIS), artificial intelligence, pattern recognition, remote sensing, supervised classification.

\section{INTRODUCTION}

A RTIFICIAL immune systems (AIS) have recently drawn increased attention from the artificial intelligence community. AIS were inspired by the human immune system and have been exploited for a wide spectrum of applications [1], [2]. Some application examples include pattern recognition [3], [4], intrusion detection [5], [6], clustering [7], and optimization [8]. An artificial immune network (AIN), which is derived from the immune network theory [9], [10], is an important and effective model of AIS. Several AIN models have been proposed and have been successfully applied to data analysis and pattern recognition, such as the multiple-valued immune network (MVIN) [11], [12], parallel distributed processing model [13], AIN (aiNet) model [2], and artificial immune net (AINE) model [7]. In spite of the successful application of AIN in several fields, few applications of current AIN models

Manuscript received December 1, 2006; revised August 5, 2007. This work was supported by the 863 High Technology Program of the People's Republic of China under Grants 2007AA12Z148 and 2007AA12Z181, by the 973 Program of the People's Republic of China under Grant 2006CB701302, and by the National Natural Science Foundation of China under Grants 40471088 and 40771139.

The authors are with the State Key Laboratory of Information Engineering in Surveying, Mapping, and Remote Sensing, Wuhan University, Wuhan 430079, China (e-mail: zlp62@public.wh.hb.cn).

Digital Object Identifier 10.1109/TGRS.2007.907739 have been reported in the area of remote sensing. This may be attributed to the high computational costs arising from current AIN models [14] that render them unfit for remote-sensing image classification. There being too many user-defined parameters in current AIN models is another obstruction. To overcome these shortcomings, this paper seeks to develop a novel algorithm based on MVIN for supervised multispectral remote-sensing image classification.

In previous works, we have successfully applied AIS to unsupervised and supervised remote-sensing image classification [15], [16]. In [15], an unsupervised artificial immune classifier (UAIC) was proposed and was successfully applied for the classification of remote-sensing imagery. The key mechanisms and concepts embodied in UAIC include antibody population evolution, clonal selection, and memory cell development. In [16], a novel classifier, i.e., the Resource-Limited Classification of Remote Sensing Images (RLCRSI), was developed for multispectral/hyperspectral classification based on the paradigm of improved AIS, i.e., a resource-limited AIS. RLCRSI is capable of performing data reduction by generating a representative set of memory cells for classification. The above algorithms were based only on the immunological properties such as clonal selection theory and immune memory and did not utilize immune network theory. Being different from the previous work, the multiple-valued immune network classifier (MVINC) not only utilizes the novel immune theory, which is the immune network theory, but also applies the multiple-valued logic theory to improve the algorithm (see also Section II for details).

A multispectral/hyperspectral image is a collection of several monochrome images of the same scene, each taken with a different sensor. Each image is referred to as a band. Multispectral/ hyperspectral images are most commonly used in image processing for remote-sensing applications. Satellites usually take several images from frequency bands in both the visual and nonvisual parts of the spectrum. Classification is a central issue in multispectral/hyperspectral image processing, and various supervised algorithms such as Parallelepiped (PP), Minimum Distance (MD), Maximum Likelihood (ML), and K-nearest neighbor (K-NN) have been designed and implemented in the past for classifying multispectral/hyperspectral data in a pixelwise manner [17], [18]. The PP classifier [17], also known as the box decision rule, is probably the simplest among the aforementioned algorithms. This algorithm employs the ranges of values within the training data to define regions within a multidimensional data space. The MD classifier [19] uses the central values of the spectral data that form the training data set to assign pixels to information categories. The K-NN algorithm [17] assumes that pixels close to each other in the 
feature space are likely to fall in the same class and assigns the classification of the majority vote among the K-NNs in the training samples to the pixel in question. As is well known, the ML classifier is a powerful classification technique based on the maximum likelihood decision rule. The rule assumes that both the training data and the classes themselves usually present multivariate normal frequency distributions [17]. To improve on classification performance, many new classifiers have also been devised for multispectral/hyperspectral remotesensing images such as artificial neural networks [20], [21], genetic algorithms (GAs) [22]-[25], support vector machines (SVMs) [26], fusion techniques [27], decision tree [28], and object-based classification [29].

Unlike the aforementioned classification algorithms, the proposed algorithm based on an MVIN is a self-learning highly robust algorithm. Specifically, the novelty of the algorithm lies in the following aspects: 1) it is a data-driven self-adaptive method as it can adjust itself to the data without any explicit specification of functional or distributional form for the underlying model; 2) it is viewed as a universal functional approximator since it can approximate any function with arbitrary accuracy; and 3) it inherits multiple-valued logic computational capability and the memory property of an MVIN and can quickly recognize the same or similar antigen at different times. The proposed algorithm has been examined with various multispectral images, and it is demonstrated that this algorithm can achieve high classification accuracy, thus providing an effective option for multispectral remote-sensing image classification.

The remainder of this paper is structured as follows. Section II gives an overview of the human immune system and the MVIN model. Section III describes the proposed method and algorithm in detail, whereas Section IV illustrates the performance of the proposed algorithm as compared to the traditional ones. Section $V$ analyzes the sensitivity of the proposed algorithm in relation to its main parameters. Finally, Section VI concludes this paper.

\section{MVIN MODEL}

The human immune system, which is a complex system of cells, molecules, and organs, symbolizes an identification mechanism capable of perceiving and combating dysfunction from our own cells and the action of exogenous infectious microorganisms. This immune system protects the body from infectious agents such as viruses, bacteria, fungi, and other parasites. Any molecule that can be recognized by the adaptive immune system is known as an antigen. The basic component of the immune system is the lymphocytes or the white blood cells. Lymphocytes exist in two forms-in B cells and T cells. Antibody molecules are synthesized and secreted by B cells, and that process is regulated by $\mathrm{T}$ cells. The $\mathrm{T}$ cells can either help or suppress the B cells' response to a stimulus [30]. In the immune response network model, a simplified system has been proposed for immunology in body fluid [11]. First, an antigen is taken in by a macrophage or some other cells with antigen processing capability. The antigen is partially digested and appears on the surface of these cells. The process and these cells are called antigen presentation and antigen-presenting cells, respectively. The antigen is recognized by T cells' receptors and activated to secrete interleukin. The interleukin is the second signal to B cells, the B cells being stimulated by the interleukin. The stimulated B cells break up and divide into antigen synthetic cells (plasma cells). In the plasma cells, antibody molecules are synthesized in great volume and secreted. If a sufficient quantity of antibody and lymphocytes are composed, we regard the immune system as being affected.

On the other hand, parts of the divided B cells are preserved as they are in the immune system and become immune memory cells. If the same antigens invade once again, the memory cells rapidly divide into plasma cells, and a large quantity of antibodies is generated in a very short period. The above process is called the secondary immune response of immune systems. After the antigen is excluded, the interleukin is secreted by suppressor $\mathrm{T}$ cells to suppress the generation of the antibody. Then, the immune response is finished [11].

There are several AIN models in the literature based on the immune response and immune network theory. The AINE model brought improvements for more general data analysis [7]. Recently, a new AIN model, namely aiNet, was proposed to solve data-clustering problems [2]. These AIN models make no distinction between the lymphocytes and their surface molecules (antibodies), and only B cells are considered in these models, that is, T cells are neglected. An MVIN model is formulated, which is different from the above model, and based on the analogy with the interaction between $\mathrm{B}$ cells and $\mathrm{T}$ cells in the immune system. Therefore, the model has a property that resembles quite well the immune response.

There are several cells involved in the immune mechanism. The principle cells involved in the interaction are antigen $(\mathrm{Ag})$, antibody $(\mathrm{Ab}), \mathrm{B}$ cells $(B)$, help $\mathrm{T}$ cells $\left(T_{H}\right)$, and suppressor $\mathrm{T}$ cells $\left(T_{S}\right)$. The model defines the input-output relation of a cell: Input $\rightarrow$ Cell $\rightarrow$ Output. The implementation of the model is detailed as follows [11].

1) Antigen (Ag), as shown in the following equation, is taken in by B cells and appears on their surface, namely antigen presentation:

$$
\mathrm{Ag} \rightarrow B \rightarrow \text { Antigen Presentation. }
$$

2) The antigen presentation, as shown in the following equation, is recognized by the help of $\mathrm{T}$ cells $T_{H}$, which secrete the interleukin $\mathrm{IL}_{+}$that activates the immune response:

$$
\text { Antigen Presentation } \rightarrow T_{H} \rightarrow \mathrm{IL}_{+} .
$$

3) The interleukin $\mathrm{IL}_{+}$, as shown in the following equation, becomes the second signal of the B cells (the B cells divide into plasma cells, then synthesize the antibodies $(\mathrm{Ab})$, and finally secrete the $\mathrm{Ab})$ :

$$
\mathrm{IL}_{+} \rightarrow B \rightarrow \mathrm{Ab}
$$

where $B$ represents both $\mathrm{B}$ cells and plasma cells.

4) If the antigen is excluded by the antibodies, then the immune response is finished. At the same time, the model must modify the concentration of antibodies. That is, the 


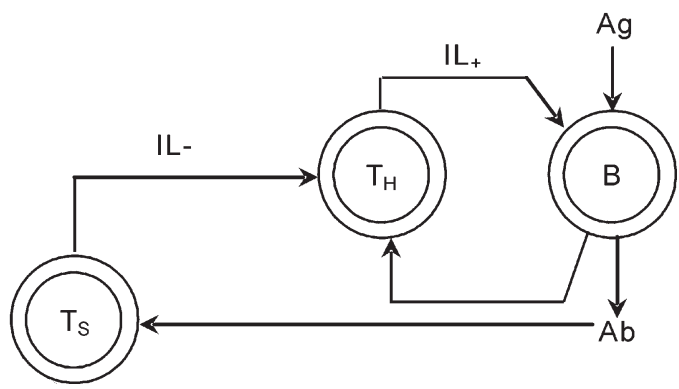

Fig. 1. Immune response network.

suppressor T cells $T_{S}$ are stimulated to secrete inhibitory interleukin $\mathrm{IL}_{-}$that suppresses the immune response. If the generation of the antibodies stops, then the immune response is finished. That is,

$$
\mathrm{Ab} \rightarrow T_{S} \rightarrow \mathrm{IL}_{-} .
$$

The immune response process mentioned above can be illustrated as an immune network, as shown in Fig. 1.

The immune system model has three important features [11]. First, if $\mathrm{Ag}$ and $\mathrm{Ab}$ are defined as an input and an output, respectively, the output is not determined by the $\mathrm{B}$ cells, but by the interaction between B cells and $T_{H}$ cells. Second, the $T_{S}$ cells that adjust the subsystem constructed by B cells and $T_{H}$ cells also play an important role in the immune system because they can suppress the immune response to avoid the infinite increase of antibodies. Third, every cell describes a set of the cells in Fig. 1; for example, Ag represents a set of the antigens.

Based on the immune response network and multiple-valued logic theory [31], the MVIN model was proposed. MVIN extends the binary representations to multiple-valued representation. Whereas a binary immune network employs binary representations for weight and memory pattern update modules, MVINs substitute multiple-valued representations for these modules. For instance, MVIN learns to classify inputs by a multiple-valued set of features from 0 to $(m-1)$ indicating the extent to which each feature is present, where $m$ represents the maximal value of multiple-valued features. The simulations show that, aside from the advantages of fewer categories, improved memory pattern, and good memory capacity, MVIN produces a stronger noise immunity than the binary network [11].

Similar to AIS (e.g., MVIN), the GA is also a heuristic algorithm. However, their underlying mechanisms and methods of evolutionary search significantly differ in terms of inspiration, vocabulary, and fundamentals. Although GA uses a vocabulary borrowed from natural genetics and is inspired by the Darwinian evolution theory, AIS makes use of the immunological terminology to describe antigen-antibody interactions and cellular evolution in immune systems. Their evolutionary search differs from the viewpoints of inspiration, vocabulary, and fundamentals [32]. In addition, AIS inherits the memory property of human immune systems to build a memory cell population and can quickly recognize the same or similar antigens at different times [2], [33].

\section{MVINC OF REMOte-SENSING IMAGERY}

A new supervised classifier based on the MVIN model, namely the MVINC, is developed in this paper for classification of multispectral imagery. A multispectral remote-sensing data set $X=\left\{x^{1}, x^{2}, \ldots, x^{N_{b}}\right\}^{T}$ through $N_{b}$ bands is observed and mapped to a finite rectangular lattice $W=\{(i, j)): 1 \leq$ $\left.i \leq N_{\text {row }}, 1 \leq j \leq N_{\text {col }}\right\}$, where $N_{\text {row }}$ and $N_{\text {col }}$ represent the row number and column number, respectively. $T$ denotes the transpose of a matrix.

Being different from the original MVIN, and to guarantee the convergence of MVINC, we add a tolerance threshold $\rho$ as the stopping criterion of the training process. In MVINC, when the maximal error between the input antigen and memory cells is in the range of $\rho$, the antigen was recognized. Otherwise, MVINC evolved until the criterion was satisfied.

The implementation of MVINC includes the following six steps:

1) selection of the regions of interest (ROI) or samples;

2) input antigen;

3) initialization of MVINC;

4) evolution of MVINC;

5) output trained MVINC (through these five steps, the trained MVINC is used to classify a multispectral remotesensing image into step 6);

6) classification.

The aforementioned steps are detailed as follows.

\section{A. Selection of ROI or Samples}

Based on the characteristics of the remote-sensing image (e.g., texture, spectra) and application purpose, ROI or samples can be selected from an image or a spectral library. In the MVINC, the training samples are represented by the set of antigens $\mathrm{AG}$, and the number of classes $C$ is equal to the number of ROI obtained in the process.

\section{B. Input Antigen}

Input pattern $\mathrm{Ag}$ is input to the B cells' group (e.g., $N$ cells). Each dot (binary or multiple-valued one) of the input pattern is input to its responding B cell layer. In MVINC, $N$ represents the dimension of input data, that is, in the task of remote-sensing image classification, it equals the number of multispectral image bands.

\section{Initialization of MVINC}

Step 1: Initialization of Antigen Presentation and Interleukin $I L_{+}$Secretion: When the input $\mathrm{Ag}$ pattern is input to its responding B cell layer, it is presented to all $T_{H}$ cells (e.g., $M$ cells). The above process is named as antigen presentation and is shown in Fig. 2. $M$ represents the number of $T_{H}$ cells. In the classification of remote-sensing imagery, the value of $M$ may be the multiple of the number of classes, such as $M=C$.

To a multiple-valued network, we can obtain $M$ in $N$-dimensional weight vectors $\boldsymbol{W}_{j}$ as follows:

$$
\boldsymbol{W}_{j}=W_{1 j}, W_{2 j}, \ldots, W_{N j}
$$




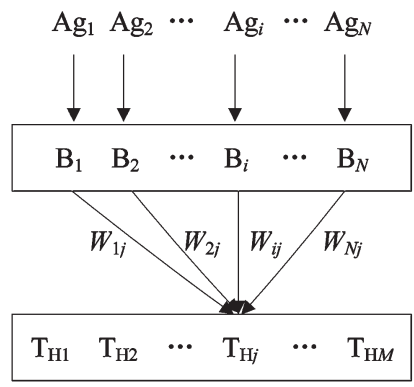

Fig. 2. Weight connections from B cell to $T_{H}$ cells.

where $j=1,2,3, \ldots, M$. The weight vector describes the stimulation level of an input $\mathrm{Ag}$ pattern to different $T_{H}$ cells. To avoid activating a cell that has never been memorized, it is necessary to initialize the weights from $\mathrm{B}$ cells to $T_{H}$ cells to be small values. This can be achieved by the following equation:

$$
W_{i j}<\frac{L}{L-1+N}
$$

where $L$ is a constant larger than $1, i=1,2, \ldots, N, N$ is the number of B cells or input pattern elements, and for remotesensing image classification, $N$ is the number of image bands. For example, for $L=2$

$$
W_{i j}(0)=\frac{1}{N}<\frac{2}{1+N} .
$$

For a multispectral remote-sensing image with six bands, $N=6$ and $W_{i j}(0)=(1 / 6)<(2 / 7)$.

Then, the $T_{H}$ cell takes the sum of the weighted input, and only the $T_{H}$ cell that receives the strongest stimulus (called antigen presentation) secretes the interleukin $\mathrm{IL}_{+}$. Namely, the $T_{H}$ cells' group can be considered as a competition network.

Step 2: Initialization of Memory Pattern: After the interleukin $\mathrm{IL}_{+}$generates the corresponding weight vector $W$, it feeds back to B cells again and obtains a feedback vector $\boldsymbol{T}_{H j}$, as shown in Fig. 3, as

$$
\boldsymbol{T}_{H j}=\left(t_{1 j}, t_{2 j}, \ldots, t_{N j}\right)
$$

where $t_{i j}=0,1,2, \ldots, m-1, i=1,2, \ldots, N, j=1,2, \ldots, M$. $m$ represents the multiple-valued features. MVINC applies the learning theory based on the set of multiple-valued features, in which different features are indicated from 0 to $m-1$. For the classification of multispectral imagery, $m$ can be replaced with the maximal gray value of the image. For instance, if $m=255$, 255 represents the maximum digital number (DN) value of the image.

The feedback vector is called the memory pattern or multiple-valued memory pattern. In MVINC, to apply MVIN to classify the remote-sensing imagery, the vector can be represented by a real number vector. In initialization, for an $m$-valued pattern, we can set

$$
t_{i j}(0)=m-1
$$

for all $i, j$.

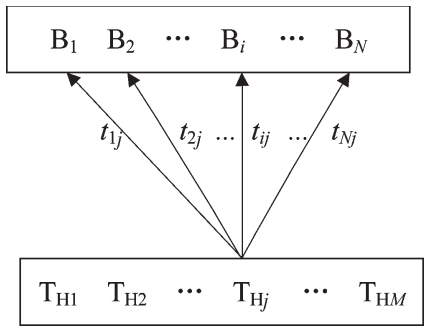

Fig. 3. Weight connections from $T_{H}$ cell to B cells.

Step 3: Antibodies Generation: After the feedback from the $T_{H}$ cell layer to the B cell layer, the errors between the input pattern vectors and the memory pattern vectors are known as the antibodies and are input to the $T_{S}$ cell layer. With a multiplevalued pattern representation, the antibodies can be described by the following equation as

$$
\mathrm{Ab}_{i}=\left|\mathrm{Ag}_{i}-t_{i j}\right|
$$

where $i=1,2, \ldots, N$.

Step 4: Stopping Criterion for the Training Procedure: In the $T_{S}$ cell layer (suppressive layer), the maximum errors (antibodies) between input pattern and memory pattern $A b_{\max }$ are obtained first, i.e.,

$$
\mathrm{Ab}_{\text {max }}=\max \left\{\mathrm{Ab}_{i}, i=1,2, \ldots, N\right\} .
$$

MVINC compares $\mathrm{Ab}_{\max }$ with the tolerance threshold $\rho$ in the following:

$$
\mathrm{Ab}_{\max }<\rho .
$$

If the value of $A b_{\max }$ is less than the tolerance threshold $\rho$, then the antigen has been recognized by MVINC. The next antigen will be input and trained by MVINC. Otherwise, the next process will be carried out.

\section{Evolution of MVINC}

If the value of $A b_{\max }$ is beyond the tolerance threshold $\rho$, then the inhibitory interleukin $\mathrm{IL}_{-}$is secreted from the $T_{S}$ cells. The inhibitory interleukin tends to suppress the $T_{H}$ cells that secreted the excitatory interleukin. Thus, a new competition in the $T_{H}$ cells' group occurs, and MVINC will evolve.

Step 1: Update of Weights From B Cell Layer to $T_{H}$ Cell Layer: The update of the $j$ th $T_{H}$ cell $\left(T_{H k}\right)$ is as follows:

$$
\begin{aligned}
W_{i j}(k+1) & =\frac{t_{i j}(k) \cdot \mathrm{Ag}_{i}}{\left\|\boldsymbol{T}_{H j}\right\| *\|\mathrm{Ag}\|+\varepsilon} \\
\|\mathrm{Ag}\| & =\sqrt{\sum_{i=1}^{N}\left|\mathrm{Ag}_{i}\right|^{2}} \\
\left\|\boldsymbol{T}_{H j}\right\| & =\sqrt{\sum_{i=1}^{N}\left|t_{i j}\right|^{2}}
\end{aligned}
$$

where $i=1,2, \ldots, N, t_{i j}$ are the feedback weights from $T_{H}$ cells to $\mathrm{B}$ cells. $\mathrm{Ag}_{i}$ is the input pattern. $k$ represents the training 


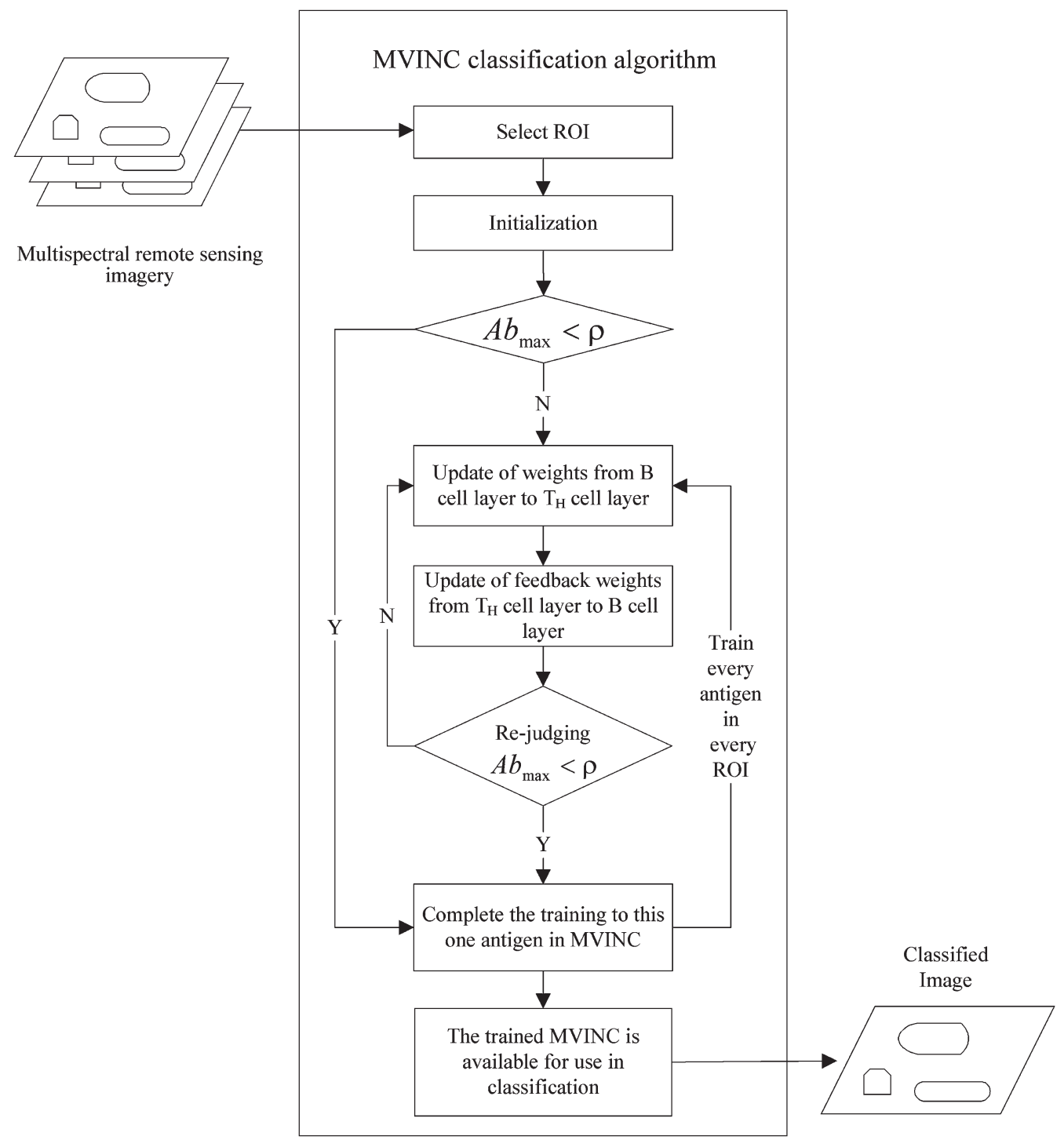

Fig. 4. Flowchart for MVINC.

or iteration number of the immune network, such that $t_{i j}(k)$ is the value of $t_{i j}$ in the $k$ th training or iteration process. $\varepsilon$ is a small positive constant, and the empirical values of $\varepsilon$ is 0.00001 . The operator $(\cdot)$ represents the inner product of the multiple-valued memory pattern vector and the input pattern vector.

Step 2: Update of Feedback Weights From $T_{H}$ Cell Layer to $B$ Cell Layer: By a real-time modified function, the feedback weights from $T_{H}$ cell layer to B cell layer can be updated using the following equation:

$$
t_{i j}(k+1)=\frac{\left|t_{i j}(k)+\mathrm{Ag}_{i}\right|}{2}
$$

where $i=1,2, \ldots, N,\lceil x\rceil$ is the smallest integer equal to or larger than $x$. Equation (12) averages the different parts between the memory pattern vector and the input pattern vector. In the other words, if an input pattern vector is classified into a category, the memory pattern vector of the category will be updated to express some features of both the memory pattern and the input pattern.
Step 3: Rejudging Stopping Criterion: Calculate the antibodies between input pattern and memory pattern. If the maximum antibodies $\mathrm{Ab}_{\max }$ is larger than a given tolerance threshold $\rho$, then the process repeats the evolutional process, i.e., Section III-D, until the stopping criterion is met. Otherwise, jump to Section III-E.

\section{E. Output Trained MVINC}

Once the aforementioned process has been carried out, training on this particular antigen is completed. The next antigen in the training set or ROI is then selected, and the training process proceeds in Section III-D. This process continues until all antigens in all ROI have been trained in the proposed algorithm. Through the five steps above, the trained MVINC is used to classify a multispectral remote-sensing image in Section III-F.

\section{F. Classification}

The classification process is as follows. First, the classified multispectral remote-sensing imagery is input to MVINC as 


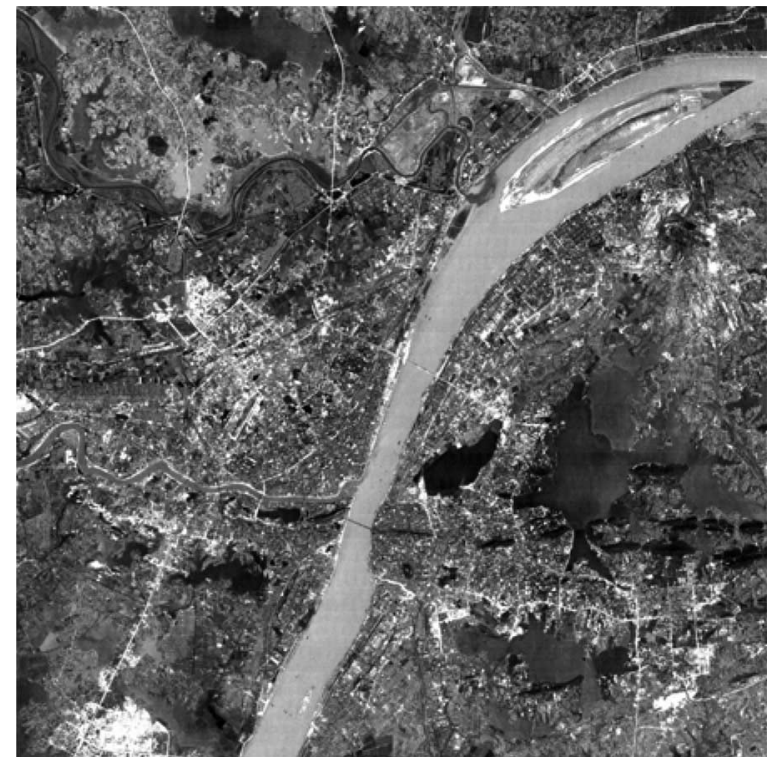

Fig. 5. Wuhan TM image, October 1998 RGB $(3,2,1)$.

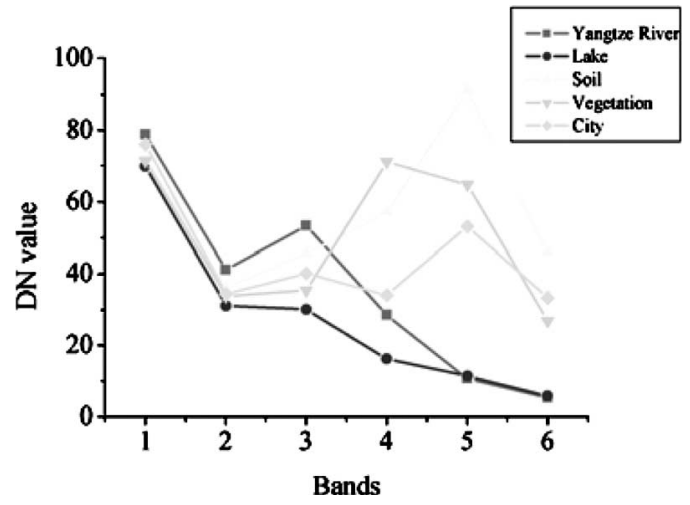

Fig. 6. Spectra of five classes.

TABLE I

LAND-COVER ClasSES AND ASSOCIATED NUMBERS OF PIXELS USED IN EXPERIMENT 1

\begin{tabular}{c|c}
\hline Class Name & Number of labeled samples \\
\hline Yangtze River & 6811 \\
\hline Lake & 10486 \\
\hline Soil & 4034 \\
\hline Vegetation & 10890 \\
\hline City & 7325 \\
\hline Total number of samples & 39546 \\
\hline
\end{tabular}

antigen. Second, the $T_{H}$ cells accept the input of weights and take the sum of the weighted input as the stimulation level. The input Ag pattern is classified to the class of the strongest $T_{H}$, which has the highest stimulation level.

The flowchart for MVINC is shown in Fig. 4.

\section{EXPERIMENTS AND ANALYSIS}

The proposed MVINC and traditional supervised algorithms were all implemented using Visual $\mathrm{C}++6.0$ and tested on different types of remote-sensing images. Two experiments were conducted to test performances. Consistent comparisons were

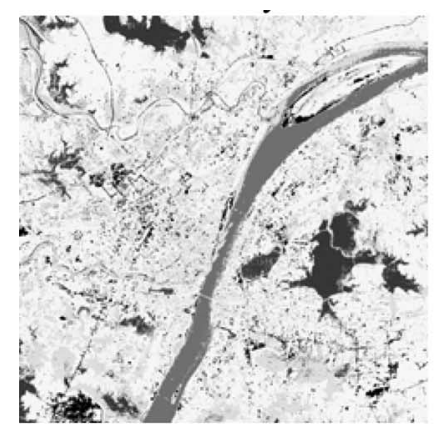

(a)

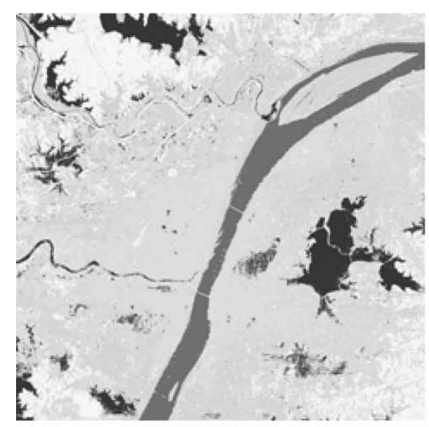

(c)

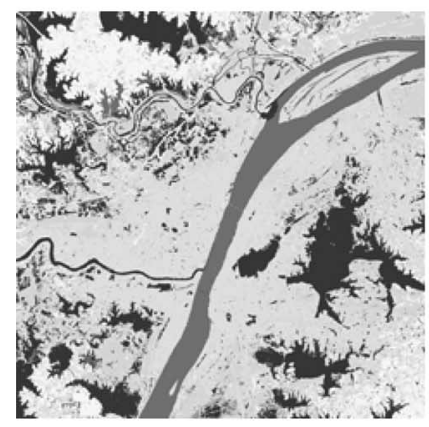

(e)

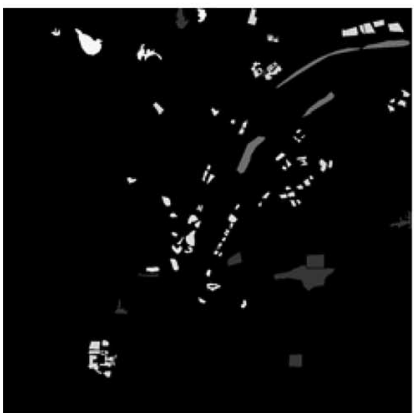

$(\mathrm{g})$

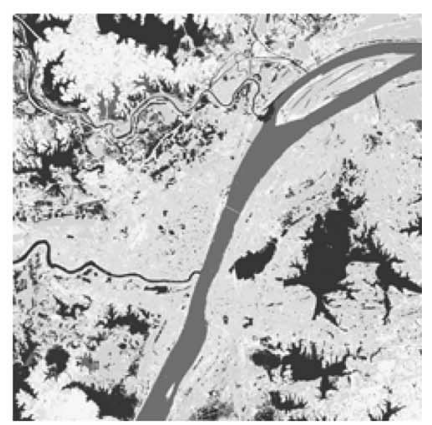

(b)

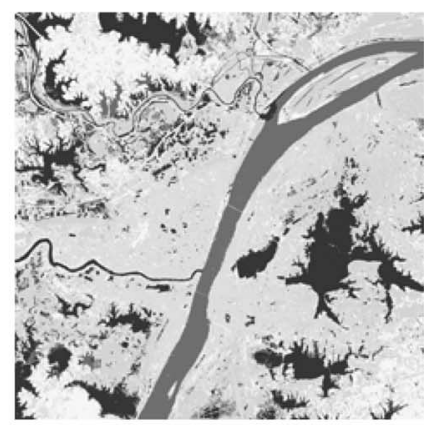

(d)

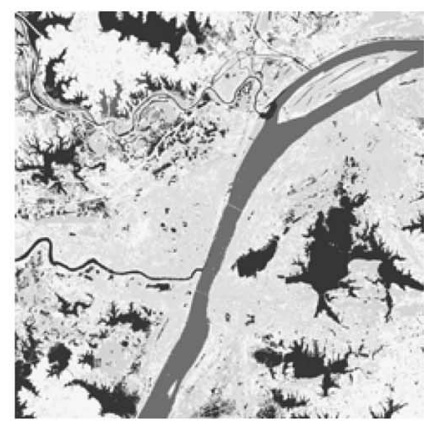

(f)

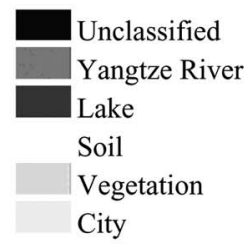

Fig. 7. Supervised classification images for Wuhan TM image. (a) PP. (b) MD. (c) ML. (d) BP (one hidden layer). (e) SVM. (f) MVINC. (g) The image for test field in Experiment 1.

also carried out between MVINC and PP, MD, ML, BackPropagation Neural Network (BP), and SVM in all the experiments. The configuration for BP (using one hidden layer) is as follows: the number of input neurons $=$ the number of input features, the number of hidden layer neurons $=$ twice the number of input layer neurons, the learning rate $=0.25$, the inertia factor $=0.95$, and the stopping rule of the training phase $=$ 1000 epochs. The options for SVM are as follows: the kernel 
TABLE II

Comparison of Six Methods of Classification In Experiment 1

\begin{tabular}{c|c|c|c|c|c|r}
\hline Class & PP & MD & ML & BP & SVM & MVINC \\
\hline Yangtze river & $99.8 \%$ & $100 \%$ & $100 \%$ & $100 \%$ & $100 \%$ & $100 \%$ \\
\hline Lake & $62 \%$ & $100 \%$ & $75.1 \%$ & $100 \%$ & $100 \%$ & $100 \%$ \\
\hline Soil & $99 \%$ & $72.1 \%$ & $81.2 \%$ & $77.9 \%$ & $79.24 \%$ & $85.2 \%$ \\
\hline Vegetation & $6.9 \%$ & $68.1 \%$ & $73.3 \%$ & $88.6 \%$ & $86.91 \%$ & $80.1 \%$ \\
\hline City & $0.4 \%$ & $56.4 \%$ & $80.6 \%$ & $66.1 \%$ & $69.5 \%$ & $84.1 \%$ \\
\hline OA & $53.62 \%$ & $79.32 \%$ & $\mathbf{8 2 . 0 4} \%$ & $\mathbf{8 6 . 5 2} \%$ & $\mathbf{8 7 . 1 3} \%$ & $\mathbf{8 9 . 8 8 \%}$ \\
\hline Kappa & $\mathbf{0 . 4 2 0 5}$ & 0.7415 & $\mathbf{0 . 7 7 5 5}$ & $\mathbf{0 . 8 3 1 5}$ & 0.8489 & 0.8625 \\
\hline
\end{tabular}

type is the radial basis function and Gamma in Kernel Function $=0.167$.

\section{A. Experiment 1: Wuhan TM Image}

The first experiment was performed using a 30-m resolution multispectral Landsat Thematic Mapper (TM) image $(1024 \times$ 1024 pixels) of Wuhan City, which was acquired on October 26, 1998 (Fig. 5). The observed image area was expected to fall into five classes: Yangtze River, Lake, Soil, Vegetation, and City. Five ROI representing the five classes were selected as training regions, and each training region had ground reference sample points. Fig. 6 shows the spectra of the five training regions. In Fig. 6, the $X$ - and $Y$-axes represent band number of the image and DN values of five classes, respectively. The list of classes and the number of labeled samples for each class are given in Table I.

In this experiment, to train and test all algorithms, the training data set and the test data set were randomly obtained from the labeled samples. The main parameters of MVINC are set as follows: $N=$ the number of bands $=6, M=$ the number of classes $=5, \quad m=$ the maximum DN values of the image $=255$, and $\rho=0.15$.

Fig. 7(a)-(e) illustrates the classification results using PP, MD, ML, BP (using one hidden layer), and SVM, respectively. Fig. 7(f) illustrates the classification result using MVINC. To evaluate the classification accuracy, a test field map is provided in Fig. 7(g) based on ground truth data obtained by field sampling. To test these algorithms, approximately half of the available samples were used as the training data set, with the other half serving as the test data set.

The visual comparisons of the six supervised classifications in Fig. 7 show varying degrees of accuracy in pixel assignment. The six classifiers have similar classification results in the Yangtze River class. However, it is hard for PP to differentiate between other classes; for example, it is hard to differentiate between vegetation and city, and there are many unknown pixels (black pixels) in its classified image [Fig. 7(a)]. MD can be confused by vegetation. ML can distinguish between vegetation and other classes better; however, it misclassifies the lake pixels to other classes. BP and SVM are competent in the classification, although some city pixels are misclassified as vegetation. However, MVINC achieves the best visual accuracy in the vegetation class and also satisfactorily performs for the soil and city classes.

Two statistics-Overall Accuracy (OA) and Kappa coefficient [17] based on the confusion matrix-are utilized to

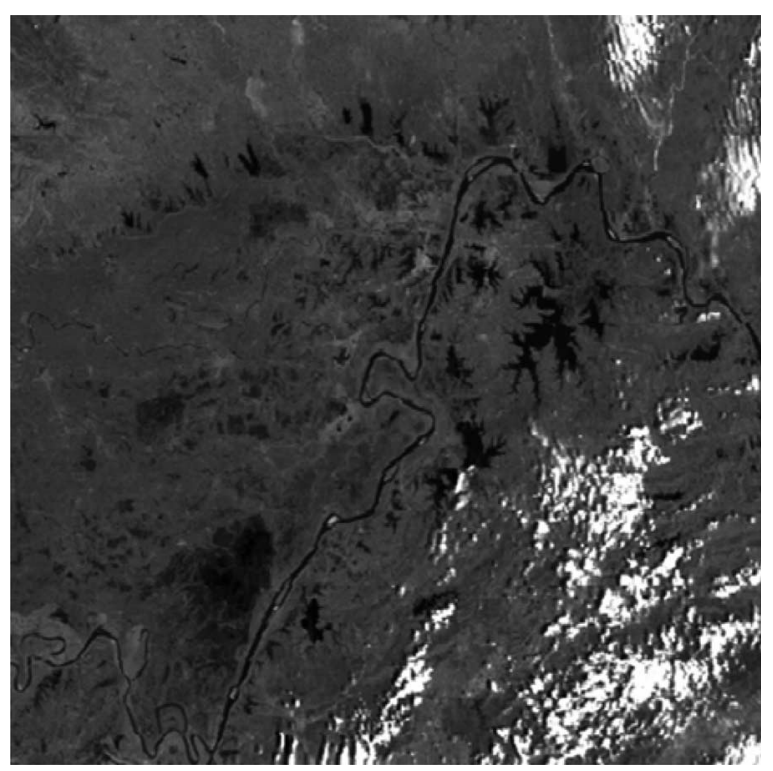

Fig. 8. Wuhan MODIS image.

evaluate the classification performances of different algorithms. Columns in a confusion matrix typically represent the reference data, and rows represent the classification data. OA is simply the sum of the pixels correctly classified (e.g., the diagonal elements), divided by the total number of samples in the comparison. The Kappa coefficient can be defined in terms of the confusion matrix as follows:

$$
\text { Kappa }=\frac{N \sum_{k=1}^{r} x_{k k}-\sum_{k=1}^{r}\left(x_{k+} \times x_{+k}\right)}{N^{2}-\sum_{k=1}^{r}\left(x_{k+} \times x_{+k}\right)}
$$

where $r$ is the number of rows in the matrix, $x_{k k}$ is the number of observations in row $i$ and column $j, x_{k+}$, and $x_{+k}$ are the marginal totals for row $i$ and column $j$, respectively, and $N$ is the total number of observations.

Table II lists the results of the comparisons between the ground truth data and classified images obtained by six classifiers: MVINC, PP, MD, ML, BP, and SVM. From Table II, it is apparent that the MVINC produces better classification results than the other classifiers. The details are as follows: MVINC exhibits the best overall classification accuracy of $89.88 \%$, i.e., the best percentage of correctly classified pixels among all the testing pixels, with a gain of $36.26 \%, 10.56 \%, 7.84 \%, 3.36 \%$, and $2.75 \%$ over the PP, MD, ML, BP, and SVM algorithms, respectively. MVINC improves the Kappa coefficient from 0.4205 to 0.8625 , an improvement of 0.442 . 


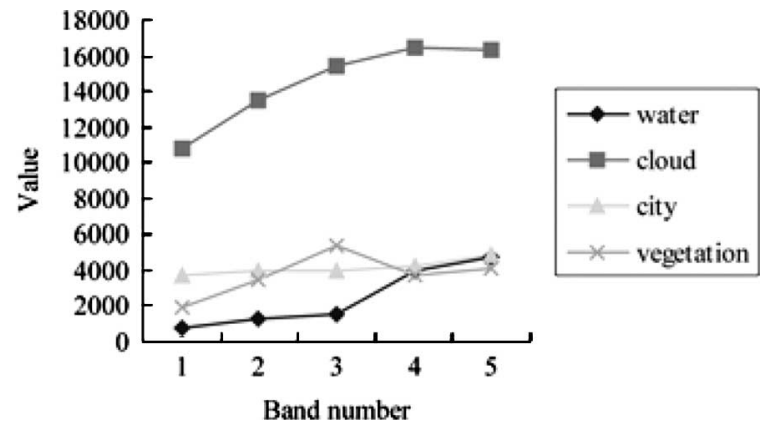

Fig. 9. Spectra of four classes.

TABLE III

LAND-COVER ClASSES AND ASSOCIATED NUMBERS of PIXELS USED IN EXPERIMENT 2

\begin{tabular}{c|c}
\hline Class Name & Number of labeled samples \\
\hline Water & 2045 \\
\hline Vegetation & 1149 \\
\hline City & 1374 \\
\hline Cloud & 1247 \\
\hline Total number of samples & 5815 \\
\hline
\end{tabular}

The main reason for the comparatively high accuracy achieved by MVINC is that ML is based on the assumption that both training data and the classes themselves display multivariate normal (Gaussian) frequency distributions [17]. However, due to the complexity of ground substances and the diversity of disturbance, data from remotely sensed images often do not strictly adhere to this rule, which, therefore, leads to the relatively poor performance. BP and SVM may achieve better accuracy; however, they require too much computational cost. In particular, the learning convergent velocity of BP is slow, and BP's accuracy is significantly influenced by the training data. In addition, the selection of learning factor and inertial factor, which are usually determined by experience, affects the convergence of the BP neural network. By contrast, MVINC is a kind of data-driven self-adaptive method, which can adjust itself to the data without any explicit specification of functional or distributional form for the underlying model. MVINC can approximate any function with arbitrary accuracy by a universal functional approximation. In addition, MVINC adopts an immune response model and multiple-valued logic theory, rendering it flexible in modeling the complex relationships between classes. These enable MVINC to achieve the best accuracy.

\section{B. Experiment 2: Wuhan MODIS Image}

In this experiment, a Moderate Resolution Imaging Spectroradiometer (MODIS) image (acquired on April 2, 2002) of an area also in Wuhan was used. The level 1B data sets include the 500-m reflectance data for channels 3, 4, 6, and 7. These four spectral channels are ordered by ascending wavelengths at $0.46-0.48,0.55-0.57,1.63-1.65$, and $2.11-$ $2.16 \mu \mathrm{m}$, respectively. The classifications were performed using four ROI, namely, water, cloud, city, and vegetation. Fig. 8 shows the experimental MODIS image, and Fig. 9 shows the spectra of the four training regions. In Fig. 9, the $X$ - and

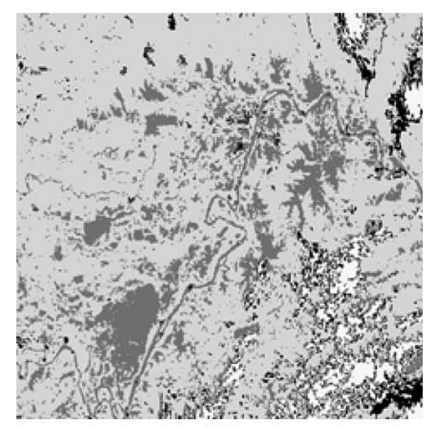

(a)

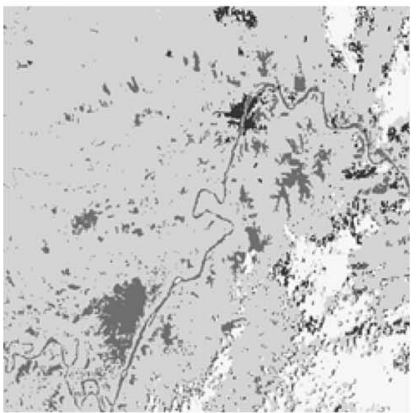

(c)

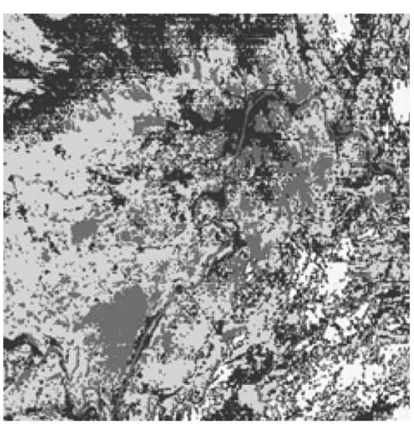

(e)

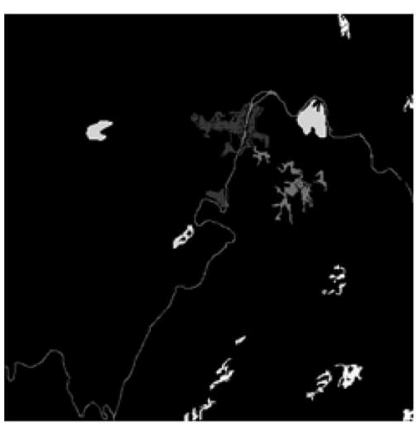

$(\mathrm{g})$

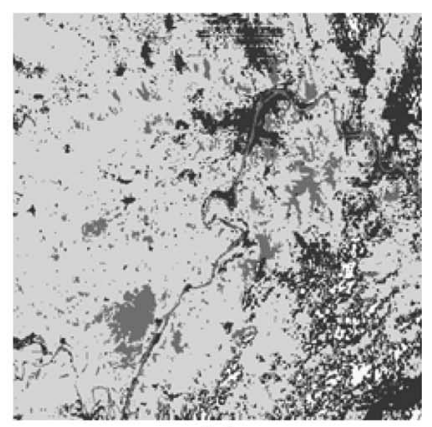

(b)

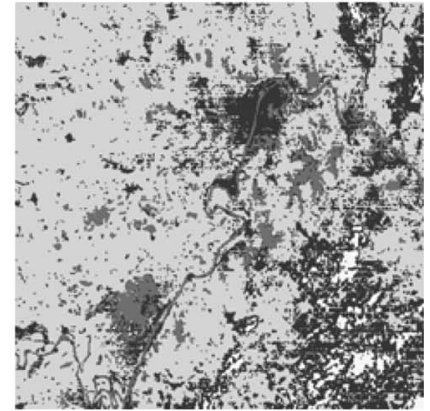

(d)

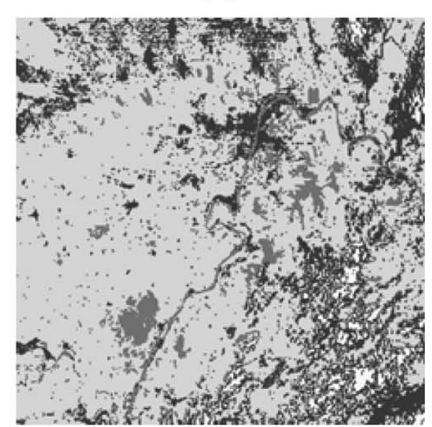

(f)
Fig. 10. Supervised classification images for Wuhan MODIS image. (a) PP. (b) MD. (c) ML. (d) BP (one hidden layer). (e) SVM. (f) MVINC. (g) The image for test field in Experiment 2.

TABLE IV

COMPARISON OF Six METHOdS OF ClASSIFICATION IN EXPERIMENT 2

\begin{tabular}{c|c|c|c|c|c|c}
\hline Accuracy & PP & MD & ML & BP & SVM & MVINC \\
\hline OA & $67.44 \%$ & $79.35 \%$ & $86.87 \%$ & $87.88 \%$ & $91.97 \%$ & $92.29 \%$ \\
\hline Kappa & 0.6122 & 0.7343 & 0.8333 & 0.8439 & 0.8908 & 0.9058 \\
\hline
\end{tabular}

$Y$-axes represent the band number of the image and the DN values of four classes, respectively. The list of classes and the number of labeled samples for each class are given in Table III. 
In the classification calculation, the main parameters of MVINC are set as follows: $N=$ the number of bands $=4$, $M=$ the number of classes $=4, m=$ the maximum $\mathrm{DN}$ values of the image, and $\rho=0.15$.

Fig. 10(a)-(f) illustrates the classification results using PP, MD, ML, BP, SVM, and MVINC, respectively. To evaluate the classification accuracy, a test field map is provided in Fig. 10(f) based on the ground truth data obtained by field sampling. To test these algorithms, approximately half of the available samples were used as the training data set, with the other half serving as the test data set.

As shown in Fig. 10, PP has many unclassified pixels. ML, MD, and BP cannot recognize the city class well. In particular, MD misclassifies many pixels of other classes to the cloud class. SVM is confused by vegetation, which is classified to other classes such as the city class. MVINC is more capable of differentiating the city class from other classes; for example, the pixels of city in the top left corner of the image are correctly classified. Therefore, MVINC can achieve better results visually. By contrast, and in addition, Table IV shows that the MVINC classifier produces better classifications than the traditional classifiers. MVINC improves the overall classification accuracy from $67.44 \%$ using PP to $92.29 \%$ (improved by $24.85 \%$ ), and the Kappa coefficient from 0.6122 to 0.9058 . These evince that MVINC is a very competent classifier, which makes it promising for remote-sensing image classification.

\section{Sensitivity Analysis}

MVINC has a parameter defined by users, namely the tolerance threshold $\rho$ as the stopping criterion of the training process. It significantly influences the convergence speed and the computational complexity of the proposed algorithm.

To analyze the effects of setting this parameter when running MVINC, the Wuhan TM image, as shown in Fig. 5, was classified using different values for this parameter. $\rho$ was assumed to have the following values: $\rho=\{0.05,0.1,0.15,0.2,0.25$, $0.3,0.35,0.4\}$. The results are presented in Fig. 11 .

As shown in Fig. 11, the smaller the $\rho$ value, the higher the OA. The reason is as follows: the smaller $\rho$ means the MVINC will tolerate fewer errors between input pattern and memory pattern. However, more computational time may be needed for the proposed algorithm to converge.

\section{CONCLUSION}

A novel supervised algorithm based on the immune network theory, namely the MVINC, was proposed in this paper. The MVINC was successfully applied for classification of remotesensing images. To guarantee the convergence of MVINC, the algorithm adds a tolerance threshold $\rho$ as the stopping criterion for the training process. In addition, MVINC is capable of performing an immune theory with memory using the multiplevalued logic theory and immune theory for classification.

A series of experiments was carried out to test the performance of MVINC using different remote-sensing images. Compared with traditional classifiers, MVINC has consistently demonstrated its better performance. In the two experiments,

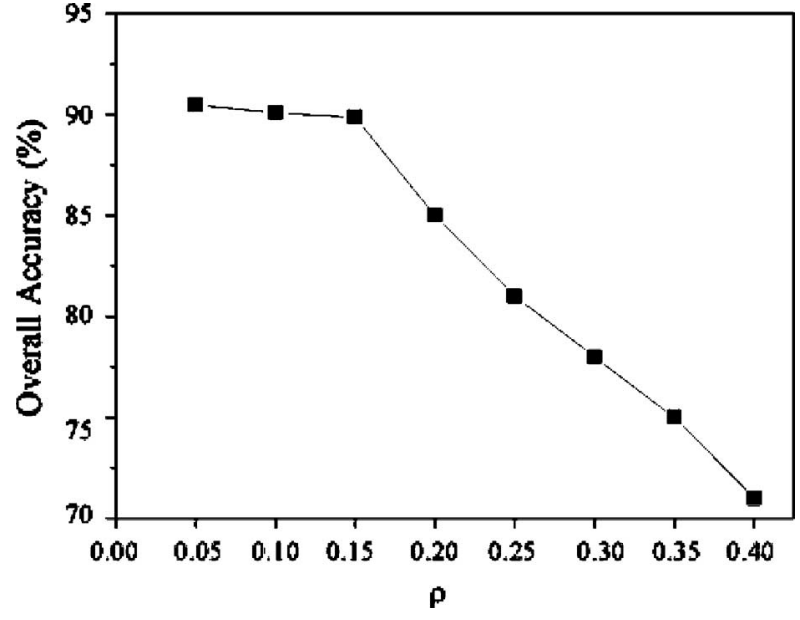

Fig. 11. OA in relation to $\rho$.

the average classification accuracy was improved from $60.53 \%$ using PP, $79.34 \%$ using MD, $84.46 \%$ using ML, $87.2 \%$ using BP, and $89.55 \%$ using SVM, to $91.09 \%$; and the Kappa coefficient improved from 0.5164 using PP, 0.7379 using MD, 0.8044 using ML, 0.8377 using BP, and 0.8699 using SVM, to 0.8842 . This evinces that the proposed method is not only able to classify multispectral remote-sensing imagery but is also a very competent classifier for data processing involving high volumes. Consequently, MVINC provides an effective option for remote-sensing image classification. In our future work, AIS and MVINC will be further explored for more extensive remote-sensing applications.

\section{REFERENCES}

[1] D. Dasgupta, Artificial Immune Systems and Their Applications. Berlin, Germany: Springer-Verlag, 1999.

[2] L. N. de Castro and J. Timmis, Artificial Immune Systems: A New Computational Intelligence Approach. London, U.K.: Springer-Verlag, 2002.

[3] J. H. Carter, "The immune system as a model for pattern recognition and classification," J. Amer. Med. Inf, Assoc., vol. 7, no. 1, pp. 28-41, Jan./Feb. 2000.

[4] A. Tarakanov and V. Skormin, "Pattern recognition by immunocomputing," in Proc. CEC, 2002, vol. 1, pp. 938-943.

[5] S. Forrest, A. S. Perelson, L. Allen, and L. Cherukuri, "Self-nonself discrimination in a computer," in Proc. IEEE Symp. Res. Security Privacy, 1994, pp. 202-212.

[6] J. Kim and P. Bentley, "Towards an artificial immune system for network intrusion detection: An investigation of clonal selection with a negative selection operator," in Proc. CEC, Seoul, Korea, 2001, vol. 2, pp. $1244-1252$.

[7] J. Timmis, M. Neal, and J. E. Hunt, "An artificial immune system for data analysis," Biosystem, vol. 55, no. 1/3, pp. 143-150, Feb. 2000.

[8] L. N. De Castro and F. J. Von Zuben, "Learning and optimization using the clonal selection principle," IEEE Trans. Evol. Comput., vol. 6, no. 3, pp. 239-250, Jun. 2002.

[9] N. K. Jerne, "Towards a network theory of the immune system," Annu. Immunol., vol. 125c, no. 1/2, pp. 51-60, Jan. 1974.

[10] A. S. Perelson, "Immune network theory," Immunol. Rev., vol. 110, pp. 536, Aug. 1989.

[11] Z. Tang, T. Yamaguchi, K. Tashima et al., "Multiple-valued immune network model and its simulations," in Proc. 27th Int Symp. MultipleValued Logic, Antigonish, NS, Canada, 1997, pp. 233-238.

[12] Z. Tang, T. Yamaguchi, K. Tashima et al., "An immune network based on biological immune response network and its immunity," IEICE Trans. Fundam., vol. J80-A, no. 11, pp. 1940-1950, 1997.

[13] F. T. Vertosick and R. H. Kelly, "Immune network theory: A role for parallel distributed processing," Immunology, vol. 66, no. 1, pp. 1-7, Jan. 1989. 
[14] O. Nasraoui, F. Gonzalez, C. Cardona, C. Rojas, and D. Dasgupta, "A scalable artificial immune system model for dynamic unsupervised learning," in Proc. GECCO, 2003, pp. 219-230.

[15] L. Zhang, Y. Zhong, and P. Li, "Applications of artificial immune systems in remote sensing image classification," in Proc. 20th Congr. Int. Soc. Photogrammetry Remote Sens., Istanbul, Turkey, Jul. 2004, vol. 35, pp. 397-401.

[16] Y. Zhong, L. Zhang, B. Huang, and P. Li, "An unsupervised artificial immune classifier for multi/hyperspectral remote sensing imagery," IEEE Trans. Geosci. Remote Sens., vol. 44, no. 2, pp. 420-431, Feb. 2006.

[17] J. B. Campbell, Introduction to Remote Sensing. London, U.K.: Taylor \& Francis, 2000.

[18] J. A. Richards and X. P. Jia, Remote Sensing Digital Image Analysis, An Introduction. Berlin, Germany: Springer-Verlag, 1999. 3rd revised and enlarged edition.

[19] R. O. Duda, P. E. Hart, and D. G. Stork, Pattern Classification, 2nd ed. Hoboken, NJ: Wiley, 2001.

[20] P. D. Heermann and N. Khazenie, "Classification of multispectral remote sensing data using a back-propagation neural network," IEEE Trans. Geosci. Remote Sens., vol. 30, no. 1, pp. 81-88, Jan. 1992.

[21] G. A. Carpenter, M. N. Gjaja, S. Gopal, and C. E. Woodcock, "ART neural networks for remote sensing: Vegetation classification from Landsat TM and terrain data," IEEE Trans. Geosci. Remote Sens., vol. 35, no. 2, pp. 308-325, Mar. 1997.

[22] B. C. K. Tso and P. M. Mather, "Classification of multisource remote sensing imagery using a genetic algorithm and Markov random fields," IEEE Trans. Geosci. Remote Sens., vol. 37, no. 3, pp. 1255-1260, May 1999.

[23] S. Bandyopadhyay and S. K. Pal, "Pixel classification using variable string genetic algorithms with chromosome differentiation," IEEE Trans. Geosci. Remote Sens., vol. 39, no. 2, pp. 303-308, Feb. 2001.

[24] U. Maulik and S. Bandyopadhyay, "Fuzzy partitioning using real-coded variable-length genetic algorithm for pixel classification," IEEE Trans. Geosci. Remote Sens., vol. 41, no. 5, pp. 1075-1081, May 2003.

[25] S. Bandyopadhyay, U. Maulik, and A. Mukhopadhyay, "Multiobjective genetic clustering for pixel classification in remote sensing imagery," IEEE Trans. Geosci. Remote Sens., vol. 45, no. 5, pp. 1506-1511, May 2007.

[26] F. Melgani and L. Bruzzone, "Classification of hyperspectral remote sensing images with support vector machines," IEEE Trans. Geosci. Remote Sens., vol. 42, no. 8, pp. 1778-1790, Aug. 2004.

[27] J. A. Benediktsson and I. Kanellopoulos, "Classification of multisource and hyperspectral data based on decision fusion," IEEE Trans. Geosci. Remote Sens., vol. 37, no. 3, pp. 1367-1377, May 1999.

[28] M. A. Friedl, C. E. Brodley, and A. H. Strahler, "Maximizing land cover classification accuracies produced by decision trees at continental to global scales," IEEE Trans. Geosci. Remote Sens., vol. 37, no. 2, pt. 2, pp. 969-977, Mar. 1999.

[29] A. K. Shackelford and C. H. Davis, "A combined fuzzy pixel-based and object-based approach for classification of high-resolution multispectral data over urban areas," IEEE Trans. Geosci. Remote Sens., vol. 41, no. 10, pt. 1, pp. 2354-2363, Oct. 2003.

[30] N. K. Jerne, "The immune system," Sci. Amer., vol. 229, no. 1, pp. 51-60, 1973.

[31] Z. Tang, Q. P. Cao, and O. Ishizuka, "A learning multiple-valued logic network: Algebra, algorithm, and applications," IEEE Trans. Comput., vol. 47, no. 2, pp. 247-251, Feb. 1998.

[32] L. N. De Castro and F. J. Von Zuben, "Artificial immune systems-part I: Basic theory and application," State Univ. Campinas, Campinas, Brazil, Tech. Rep.-RT DCA 01/99, 1999.

[33] J. S. Chun, H. K. Jung, and S. Y. Hahn, "A study on comparison of optimization performances between immune algorithm and other heuristic algorithms," IEEE Trans. Magn., vol. 34, no. 5, pp. 2972-2975, Sep. 1998

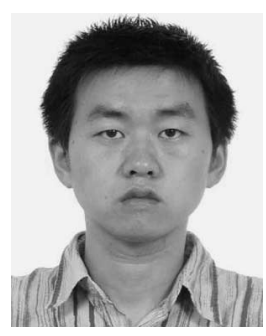

Yanfei Zhong received the B.S. degree in information engineering from Wuhan University, Wuhan, China, in 2002, and the Ph.D. degree in photogrammetry and remote sensing from Wuhan University, Wuhan, China, in 2007.

$\mathrm{He}$ is with the State Key Laboratory of Information Engineering in Surveying, Mapping, and Remote Sensing, Wuhan University. His current research interests focus on multi- and hyperspectral image processing, artificial immune systems, and pattern recognition.

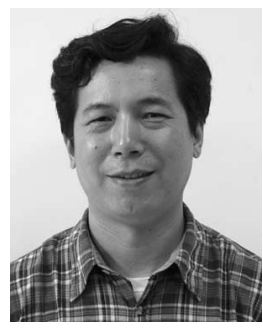

Liangpei Zhang (M'06) received the B.S. degree in physics from Hunan Normal University, ChangSha, China, in 1982, the M.S. degree in optics from the Xi' an Institute of Optics and Precision Mechanics, Chinese Academy of Sciences, Xi'an, China, in 1988 , and the Ph.D. degree in photogrammetry and remote sensing from Wuhan University, Wuhan, China, in 1998.

From 1997 to 2000, he was a Professor with the School of the Land Sciences, Wuhan University. Since August 2000, he has been with the State Key Laboratory of Information Engineering in Surveying, Mapping, and Remote Sensing, Wuhan University as a Professor and Head of the Remote Sensing Section. He has published more than 120 technical papers. His research interests include hyperspectral remote sensing, high-resolution remote sensing, image processing, and artificial intelligence.

Dr. Zhang has served as Cochair of the SPIE Series Conferences on Multispectral Image Processing and Pattern Recognition (MIPPR) and the Conference on Asia Remote Sensing in 1999, Editor of the MIPPR01, MIPPR05, and Geoinformatics Symposiums, Associate Editor of the Geospatial Information Science Journal and Chinese National Committee for the International Geosphere-Biosphere Programme, and an executive member of the China Society of Image and Graphics.

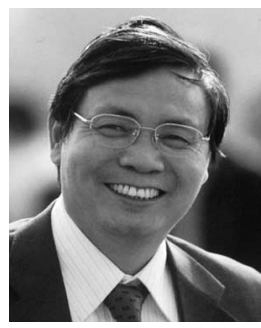

Jianya Gong received the B.S. degree in surveying and mapping from East China Geology College, Jiangxi, in 1982, and the Ph.D. degree in photogrammetry and remote sensing from Wuhan Technical University of Surveying and Mapping, Wuhan, China, in 1992.

Since 1994, he has been a Professor with the State Key Laboratory of Information Engineering in Surveying, Mapping, and Remote Sensing, Wuhan University, Wuhan, China. His research interests include photogrammetry, remote sensing, and geographic information systems.

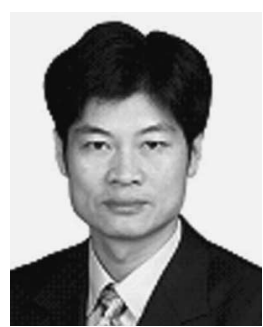

Pingxiang Li (M'06) received the B.S., M.S., and $\mathrm{Ph} . \mathrm{D}$. degrees in photogrammetry and remote sensing from Wuhan University, Wuhan, China, in 1986, 1994, and 2003, respectively.

Since 2002, he has been a Professor with the State Key Laboratory of Information Engineering in Surveying, Mapping, and Remote Sensing, Wuhan University. His research interests include photogrammetry and synthetic aperture radar image processing. 